Recepción: 17 / 04 / 2018

Aceptación: 19 / 05 / 2018

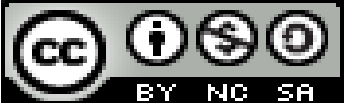

Ciencias económicas y empresariales

Publicación: 01 / 08 / 2018

Artículo de investigación

\title{
Análisis de la cultura tributaria en el personal de servidores públicos de la escuela de calificación y de perfeccionamiento
}
Analysis of tax culture in the staff of public servants of qualification and training school

\section{Análise da cultura tributária no pessoal dos funcionários públicos da escola de classificação e melhoria}

\author{
Jinsop E. Gamboa-Poveda I \\ jgamboap@unemi.edu.ec \\ Segundo R. Zea-Jiménez II \\ szeaj@unemi.edu.ec \\ Xavier F. Ortega-Haro III \\ xortegah@unemi.edu.ec \\ Hugo E. Hidalgo-Hidalgo VI \\ hhidalgoh@unemi.edu.ec
}

Correspondencia: jgamboap@unemi.edu.ec

\footnotetext{
I Magister en Administración y Dirección de Empresas, Contador Público Autorizado Universidad Estatal de Milagro, Milagro, Ecuador.

II Máster en Administración de Empresas M.B.A, Ingeniero Comercial, Universidad Estatal de Milagro, Milagro, Ecuador.

${ }^{\text {III }}$ Magister en Contabilidad y Auditoría-Contador Público, Ingeniero Financiero, Universidad Estatal de Milagro, Milagro, Ecuador

${ }^{\mathrm{VI}}$ Magister en Administración y Dirección de Empresas, Diploma Superior en Diseño Curricular por Competencias, Tecnólogo en Marketing y Ventas, Universidad Estatal de Milagro, Milagro, Ecuador.
} 


\title{
Resumen
}

El presente artículo tiene como objetivo analizar las posibles mejoras para fomentar una cultura tributaria, concientizando las obligaciones tributarias que tienen que cumplir los servidores públicos de la Escuela de Calificación y de Perfeccionamiento, ESCAPE, de tal manera que el tributo lo visualice como un deber más y conozcan todo lo relacionado en temas básicos tributarios para lograr su acertada participación social y presupuestaria en el estado. La metodología fue descriptiva y de campo, la población la constituyeron 54 encuestados de un curso de maros a cabos que pertenecen a la división Delta y Foxtrot. El instrumento fue un cuestionario y la técnica la encuesta. Los resultados fueron presentados en tablas con sus correspondientes gráficos, a través de una estadística de frecuencias tanto absolutas como relativas, y el total de encuestados. Entre las conclusiones se pudo evidenciar que existe un desconocimiento en la norma tributaria y las sanciones de las mismas, lo cual implicaría futuras deudas pendientes.

Palabras clave: cultura tributaria; obligaciones tributarias; servidores públicos.

\begin{abstract}
This article aims to analyse possible improvements to foster a culture of tax, raising awareness of the tax obligations that have to meet public servants of qualification and training, ESCAPE school, of so that tribute displayed it as one duty more and learn about everything related to basic tax issues to achieve their successful social and budgetary participation in the State. The methodology was descriptive and field, population constituted 54 surveyed a course of maros to cabos belonging to the division Delta and Foxtrot. The instrument was a questionnaire and the survey technique. The results were presented in tables with their corresponding graphics, through a statistic of both absolute and relative frequencies, and the total number of respondents. Among the findings demonstrate that there is a lack of knowledge on the tax standard and the same sanctions, which would mean future outstanding debts.
\end{abstract}

Keywords: tax; tax liabilities; public servants. 


\section{Resumo}

Este artigo tem como objetivo analisar as possíveis melhorias a promover a cultura fiscal, a sensibilização das obrigações fiscais que eles têm para atender servidores públicos Qualifying School e Melhoria, escape, para que o imposto visualizá-lo como um dever mais e saber tudo relacionado a questões tributárias básicas para alcançar sua participação social e orçamentária bem-sucedida no estado. A metodologia foi descritiva e de campo, a população foi composta por 54 respondentes de um curso de maros a cabos pertencente à divisão Delta e Foxtrot. O instrumento foi um questionário e a técnica a pesquisa. Os resultados foram apresentados em tabelas com seus gráficos correspondentes, através de estatísticas de frequências absolutas e relativas e do número total de respondentes. Entre as conclusões, pode-se demonstrar que há falta de conhecimento sobre a norma tributária e suas penalidades, o que implicaria em futuras dívidas pendentes.

Palavras chave: cultura tributária; obrigações tributárias; servidores públicos.

\section{Introducción}

El presente artículo es realizado de acuerdo a la realidad que enfrentan hoy en día los servidores públicos de la Escuela de Calificación y Perfeccionamiento de la Armada (ESCAPE), debido a que se detectó escasos conocimientos tributarios, y beneficios que pueden obtener al tributar correctamente y a realizar a tiempo el pago de impuestos.

Los cambios constantes de las reformas tributarias y los avances tecnológicos son los motivos por el cual los servidores públicos de la ESCAPE se desmotivan de aprender por la complejidad de las normas tributarias o por no poder interpretar o desconocer la Ley; así también como la edad, carga de trabajo, situación socio-económica.

Uno de los objetivos de la formación de la cultura tributaria, según Amaguaya (2016) debe ser, precisamente, romper ese círculo vicioso y hacer conciencia de que la tributación no sólo es una obligación legal, sino un deber de cada persona ante la sociedad. Además, se le debe convencer de que cumplir con tal responsabilidad le confiere la autoridad moral necesaria para exigir al Gobierno que haga un uso correcto y transparente de los recursos públicos 
Todo esto sirve para que este personal realice correctamente sus declaraciones de manera oportuna, evitando ser sancionado o determinado por la Administración Tributaria. En virtud de lo expuesto en este artículo se analizan las posibles mejoras para fomentar una cultura tributaria, concientizando las obligaciones tributarias que tienen que cumplir los servidores públicos de la Escuela de Calificación y de Perfeccionamiento. A tal efecto, servirá de apoyo para que los contribuyentes realicen a tiempo y de forma correcta sus declaraciones, impidiendo ser sancionado o cancelar multas e intereses por presentación de declaraciones tardías.

\section{Desarrollo}

Cultura tributaria. Son acciones que tiene como objetivo dar a conocer la importancia de la tributación para el crecimiento y el desarrollo tanto económico como ético de la sociedad, porque son numerosos los beneficios sociales alcanzados mediante la tributación. (Centro Interamericano de Administraciones Tributarias - CIAT, 2011, pág. 8).

Evasión y elusión tributaria. La evasión es una violación de la ley cuando un contribuyente se abstiene de reportar ingresos del trabajo o capital que son parte de la base imponible para pagar impuestos. Por otro lado, elusión es la búsqueda de modos legales que permiten cancelar menos impuestos de los requeridos. La fuga tributaria, en cambio, se da cuando un negocio cambia de localización hacia paraísos fiscales para evitar el pago de tributos al Estado. (Camarero L., Pino J. y Mañas B., 2015, pág. 99)

Impuesto a la Renta (IR). En su Art. 1 la Ley de Régimen Tributario establece el objeto del impuesto, y expresa: "Establéese el impuesto a la renta global que obtengan las personas naturales, las sucesiones indivisas y las sociedades nacionales o extranjeras, de acuerdo con las disposiciones de la presente Ley” (Ley de Régimen Tributario Interno. (2010). Ecuador)

\section{Régimen Impositivo Simplificado Ecuatoriano}

El Régimen Impositivo Simplificado Ecuatoriano (RISE), tiene como objetivo principal, la simplificación del pago de los impuestos de un sector de contribuyentes y se basa en la cancelación de cuotas mensuales. Este sistema, reemplaza el pago del IVA y del Impuesto a la Renta a través del pago de dicha cuota, su implementación tiene como finalidad promover la cultura tributaria en el país y se encuentra vigente desde el 1 de agosto de 2008. 
Pueden acceder a él, de manera voluntaria, las personas naturales que cumplan con las condiciones establecidas en la Ley de Régimen Tributario Interno. (Código Tributario, 2013. Ecuador).

En materia tributaria existe una gran cantidad de contribuyentes, y entre ellos se encuentran una parte de los servidores públicos de la ESCAPE, que no conocen que se pueden beneficiar del pago de sus impuestos de ya que no solo les afecta a los demás sino también a ellos, se repercute en que el país no va a tener recursos para tener una buena infraestructura vial, salud, recreación a los que se tiene derecho y no disfrutar de esos beneficios.

\section{Metodología}

La metodología que se utilizó en este trabajo fue descriptiva y de campo, se realizaron encuestas para establecer el nivel de conocimientos tributarios de los servidores públicos de ESCAPE, se escogió como muestra a un curso de mando y liderazgo de los señores sargentos de diferentes especialidades y un curso de maros a cabos, dichas encuestas se las realizó en el mes de junio del 2018, utilizando una muestra significativa de 54 encuestados, que corresponden a la división Delta y Foxtrot. Para el desarrollo de la información se utilizó la herramienta de Excel para la tabulación de los datos obtenidos en las encuestas, mediante la aplicación de la tabla dinámica. Los resultados fueron presentados en tablas con sus correspondientes gráficos, a través de una estadística de frecuencias tanto absolutas como relativas, y el total de encuestados.

\section{Resultados}

El análisis y la discusión de los resultados se presentan en tablas con sus respectivos gráficos. En la que se detalla la relación entre la información y su representación porcentual.

Tabla 1: Conocimiento de sanciones

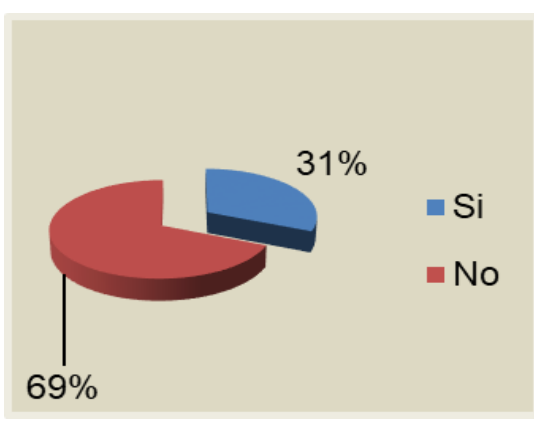

Fuente: Elaboración propia 


\begin{tabular}{|l|c|c|}
\hline $\begin{array}{c}\text { 1. ¿Conoce las } \\
\text { sanciones a las que } \\
\text { puede estar afecto en } \\
\text { caso no pague sus } \\
\text { tributos? }\end{array}$ & $\begin{array}{c}\text { Total } \\
\text { General }\end{array}$ & Porcentaje \\
\hline $\mathrm{Si}$ & 17 & $31 \%$ \\
\hline No & 37 & $69 \%$ \\
\hline TOTAL & $\mathbf{5 4}$ & $\mathbf{1 0 0 \%}$ \\
\hline
\end{tabular}

Figura 1: Conocimientos de sanciones

Fuente: Elaboración propia

Los encuestados respondieron que el $69 \%$ desconoce del tipo de sanciones que se puede conllevar el no tributar correctamente, mientras que el $31 \%$ tiene conocimiento de las consecuencias que conlleva el no tributar.

Tabla 2: Conocimientos de derechos y obligaciones.

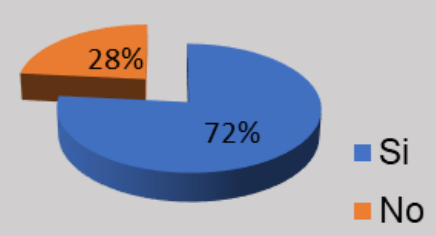

Fuente: Elaboración propia

\begin{tabular}{|l|c|r|}
\hline $\begin{array}{c}\text { 2. ¿Conoce } \\
\text { usted sus } \\
\text { derechos y } \\
\text { obligaciones } \\
\text { tributarias? }\end{array}$ & $\begin{array}{c}\text { Total } \\
\text { General }\end{array}$ & Porcentaje \\
\hline $\mathrm{Si}$ & 15 & $28 \%$ \\
\hline No & 39 & $72 \%$ \\
\hline TOTAL & $\mathbf{5 4}$ & $\mathbf{1 0 0 \%}$ \\
\hline
\end{tabular}

Figura 2: Conocimientos de derechos y obligaciones 


\begin{tabular}{|l|c|c|}
\hline $\begin{array}{c}\text { 3. Califique usted } \\
\text { cuál es el grado } \\
\text { de necesidad de } \\
\text { fomentar la } \\
\text { cultura tributaria } \\
\text { en ESCAPE }\end{array}$ & $\begin{array}{c}\text { Total } \\
\text { General }\end{array}$ & Porcentaje \\
\hline $\mathrm{Si}$ & 39 & $72 \%$ \\
\hline No & 15 & $28 \%$ \\
\hline TOTAL & $\mathbf{5 4}$ & $\mathbf{1 0 0 \%}$ \\
\hline
\end{tabular}

Fuente: Elaboración propia

Figura 3: Grado de necesidad de fomentar cultura tributaria.

El $72 \%$ de los encuestados desconoce sus derechos y obligaciones en temas tributarios, mientras que $28 \%$ dice conocerlos.
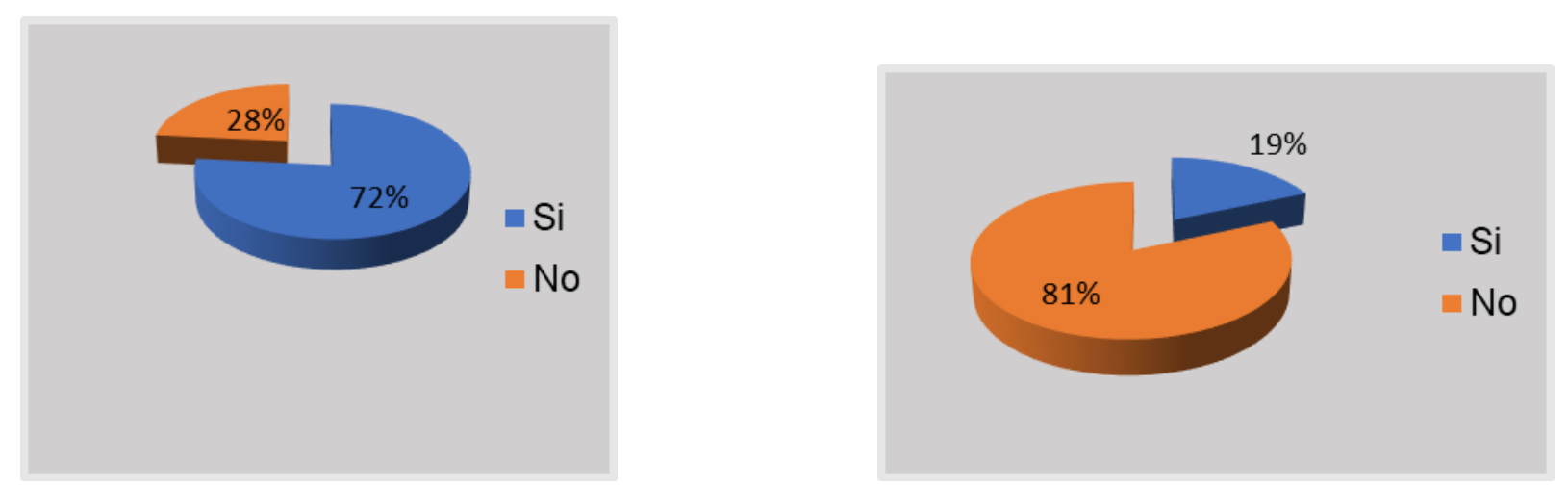

Fuente: Elaboración propia.

El $72 \%$ sienten la necesidad de que se fomente una cultura tributaria en nuestro entorno, mientras que la minoría es de un $28 \%$.

Tabla 4: Asistencia a charlas dictadas por el Sri

\begin{tabular}{|l|c|c|}
\hline $\begin{array}{c}\text { 4. iAlguna vez ha } \\
\text { asistido a charlas } \\
\text { dictadas por el SRI } \\
\text { respecto a } \\
\text { información } \\
\text { tributaria, que le ha } \\
\text { sido de mucha } \\
\text { ayuda? }\end{array}$ & $\begin{array}{c}\text { Total } \\
\text { General }\end{array}$ & Porcentaje \\
\hline Si & 10 & $19 \%$ \\
\hline No & 44 & $81 \%$ \\
\hline TOTAL & $\mathbf{5 4}$ & $\mathbf{1 0 0 \%}$ \\
\hline
\end{tabular}




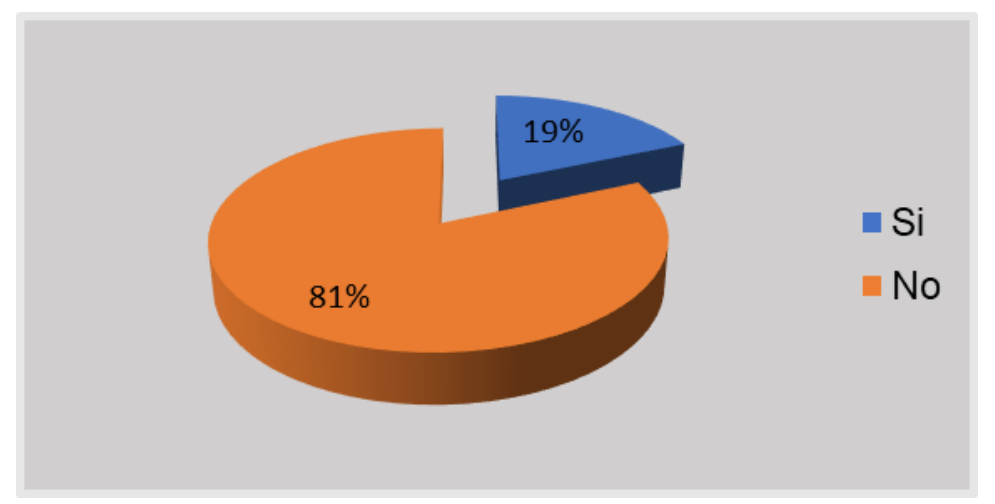

Fuente: Elaboración propia.

Figura 4: Asistencia a charlas dictadas por el Sri.

La mayoría de los encuestados afirma que el $81 \%$ ha asistido a charlas dictadas por el SRI, mientras que en su minoría el $19 \%$ no ha recibido ese tipo de charla.

Tabla 5: Atraso en declaraciones.

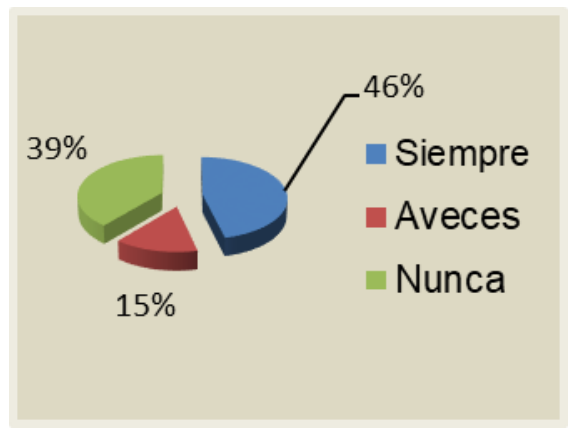

Fuente: Elaboración propia.

\begin{tabular}{|l|c|c|}
\hline $\begin{array}{c}\text { 5. ¿Con qué frecuencia } \\
\text { considera Usted que se } \\
\text { atrasa en sus } \\
\text { declaraciones? }\end{array}$ & $\begin{array}{c}\text { Total } \\
\text { General }\end{array}$ & Porcentaje \\
\hline Siempre & 25 & $46 \%$ \\
\hline A veces & 8 & $15 \%$ \\
\hline Nunca & 21 & $39 \%$ \\
\hline TOTAL & $\mathbf{5 4}$ & $\mathbf{1 0 0 \%}$ \\
\hline
\end{tabular}

Figura 5: Atraso en declaraciones.

Fuente: Elaboración propia. 
Hay un alto porcentaje de los encuestados que admiten atrasos en sus declaraciones es un $46 \%$, mientras el $15 \%$ suele olvidarse y cae en atrasos, aunque que el 39\% nunca se ha atrasado en sus obligaciones.

Tabla 6: Implementación de programas para incrementar la cultura tributaria.

\begin{tabular}{|c|c|c|}
\hline $\begin{array}{c}\text { 6. ¿Considera usted que } \\
\text { la implementación de } \\
\text { programas como una } \\
\text { feria tributaria o una } \\
\text { adecuada capacitación } \\
\text { tributaria ayudara a } \\
\text { incrementar la cultura } \\
\text { tributaria en ESCAPE? }\end{array}$ & $\begin{array}{c}\text { Total } \\
\text { General }\end{array}$ & Porcentaje \\
\hline Si & 54 & $100 \%$ \\
\hline No & 0 & $0 \%$ \\
\hline TOTAL & $\mathbf{5 4}$ & $\mathbf{1 0 0 \%}$ \\
\hline
\end{tabular}

Fuente: Elaboración propia

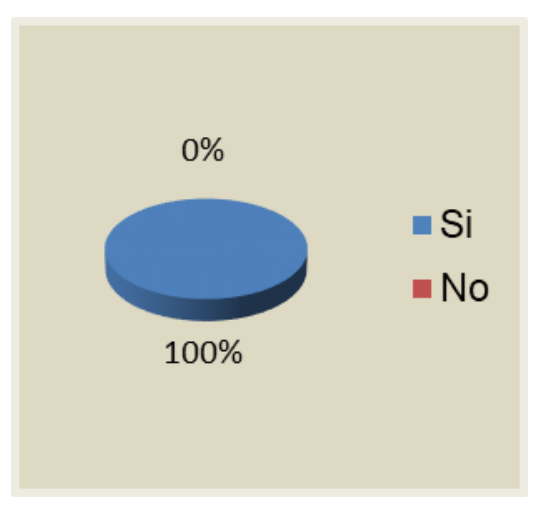

Figura 6: Implementación de programas para incrementar la cultura tributaria

Fuente: Elaboración propia

En esta pregunta encontramos la aceptación total y buena acogida que tendría el desarrollo de programas que conlleve a la cultura tributaria. 
Tabla 1: Dificultad para comprender temas tributario.

\begin{tabular}{|l|c|c|}
\hline $\begin{array}{c}\text { 7. ¿Qué motivo } \\
\text { considera usted que } \\
\text { dificulta comprender los } \\
\text { temas tributarios? }\end{array}$ & $\begin{array}{c}\text { Total } \\
\text { General }\end{array}$ & Porcentaje \\
\hline Falta de información & 25 & $46 \%$ \\
\hline Cambios constantes & 7 & $13 \%$ \\
\hline Complejidad de la ley & 22 & $41 \%$ \\
\hline TOTAL & $\mathbf{5 4}$ & $\mathbf{1 0 0 \%}$ \\
\hline
\end{tabular}

Fuente: Elaboración propia.

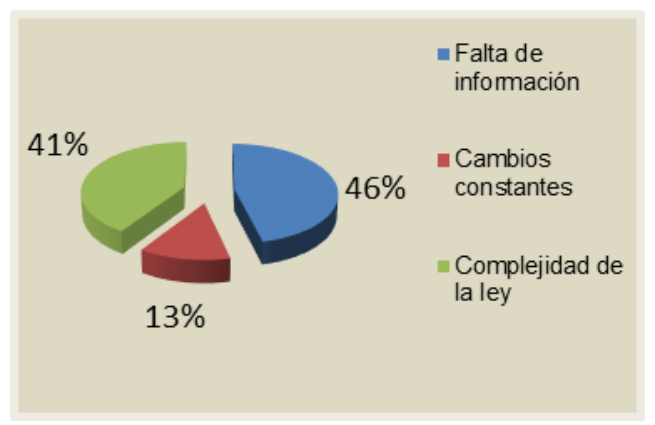

Figura 1: Dificultad para comprender temas tributarios.

Fuente: Elaboración propia.

Es muy notable que la falta de información es el motivo que conlleva a la dificultad de entender temas tributarios obtuvo un $46 \%$, aunque no es mucha la diferencia de la complejidad de las leyes, y en su menoría los cambios constantes tienen un 7\% de selección.

\section{Conclusiones}

Existe un desconocimiento en la norma tributaria y las sanciones de las mismas, lo cual implicaría futuras deudas pendientes. La poca cultura tributaria hace que desconozcan de los derechos y obligaciones tributarias y esto afecta directamente a su economía, pagando valores altos por la rapidez del servicio. Los cambios constantes de las leyes y la complejidad del tema tributario hacen que los servidores públicos muestren poco interés en aprender más del tributo.

Fomentando la cultura tributaria romperíamos ese círculo vicioso y haríamos conciencia de que la tributación no sólo es una obligación legal, sino un deber de cada persona ante la sociedad. 


\section{Referencias Bibliográficas}

Amaguaya, J. Moreira, L. (2016). "La cultura tributaria y las obligaciones fiscales de los negocios informales de Guayaquil”. Universidad de Guayaquil, Guayaquil.

Camarero L, Pino J y Mañas B. (2015) Evolución de la cultura tributaria, coyuntura

económica y expectativas vitales: Un estudio longitudinal. CIS- Centro de Investigaciones Sociológicas

Centro Interamericano de Administraciones Tributarias - CIAT (2011) La Moral Tributaria como factor determinante en el mejoramiento de la eficacia de la Administración Tributaria, Ecuador.

Ley de Régimen Tributario Interno. (2010). Ecuador. Recuperado el 26 de Julio de 2015, en línea en http://www.sri.gob.ec/web/guest/incentivos-ybeneficios. 